\title{
Self-Biased Differential Rectifier with Enhanced Dynamic Range for Wireless Powering
}

\author{
Mahmoud H. Ouda, Student Member, IEEE, Waleed Khalil, Senior Member, IEEE \\ and Khaled N. Salama, Senior Member, IEEE
}

\begin{abstract}
A self-biased, cross-coupled, differential rectifier is proposed with enhanced power-conversion efficiency over an extended range of input power. A prototype is designed for UHF $433 \mathrm{MHz}$ RF power-harvesting applications and is implemented using $0.18 \mu \mathrm{m}$ CMOS technology. The proposed rectifier architecture is compared to the conventional cross-coupled rectifier. It demonstrates an improvement of more than $40 \%$ in the rectifier power conversion efficiency (PCE) and an input power range extension of more than $50 \%$ relative to the conventional cross-

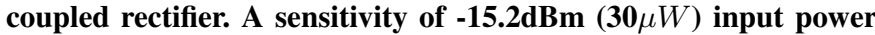
for $1 \mathrm{~V}$ output voltage and a peak power-conversion efficiency of $65 \%$ are achieved for a $50 k \Omega$ load.
\end{abstract}

Index Terms-AC-to-DC power converter, adaptive, energy harvesting, rectifier, RFID, self-biased, wireless powering.

\section{INTRODUCTION}

W IRELESS power transfer (WPT) applications range from powering battery-less wireless sensors [1], RFIDs [2], and implantable devices [3]-[5], to the higher scale of space-based solar cells and wirelessly charged electric vehicles. It utilizes various frequency bands starting from low- $\mathrm{MHz}$ for low-power implants [4], [5] to UHF RFID tags [2], including the $433 \mathrm{MHz}$ industrial, scientific, and medical (ISM) band [6] that is used for license-free communication, underwater wireless-sensor networks [7], and automotive applications such as battery-less tire-pressure monitoring systems [8]. Among this myriad of battery-less devices, the wireless power receiver is considered the linchpin for any given WPT system.

As shown in Fig.1, RF-to-DC power-converter is the core of any wireless power receiver that supplies usable DC power out of the incoming RF power to the antenna. Recently, RF-to-DC power converter is used for recycling wasted RF power in hybrid duplexers and enhancing the power amplifiers efficiency [9]. The RF-to-DC power converter performance is evaluated in terms of two main parameters: a) sensitivity, which defines the minimum input RF power required to generate a specific DC output voltage, and b) power-conversion efficiency (PCE), which is the ratio of the usable output DC power, $P_{o}$, to the input RF power, $P_{i}$, and can be expressed by (1):

$$
P C E=\frac{P_{o}}{P_{i}}=\frac{P_{o}}{P_{o}+P_{\text {diss }}+P_{\text {rvs }}},
$$

Where the input power, $P_{i}$, equals the summation of the output DC power, $P_{o}$, the dissipated power in rectifying devices,

M. H. Ouda and K. N. Salama are with King Abdullah University of Science and Technology (KAUST), Thuwal 23955-6900, Saudi Arabia (email: mahmoud.ouda@kaust.edu.sa; khaled.salama@kaust.edu.sa).

W. Khalil is with ElectroScience Laboratory, The Ohio State University, Columbus, OH 43212, USA (e-mail: khalil@ece.osu.edu).

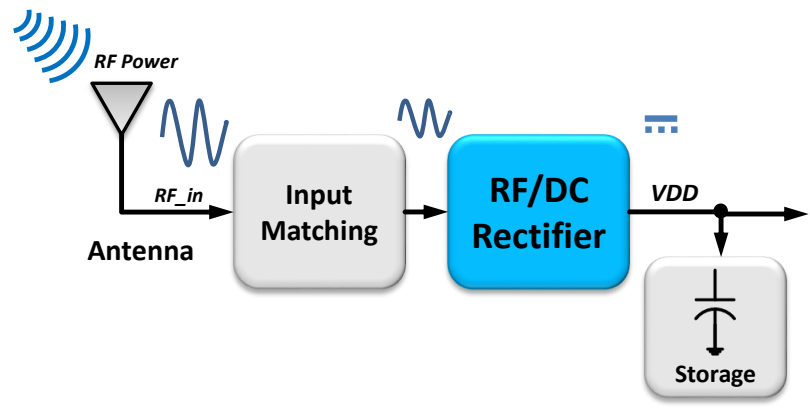

Fig. 1. Block diagram of wireless power receiver.

$P_{d i s s}$, and the reverse-leakage power, $P_{r v s}$, which is caused by the non-idealities in the reverse characteristic of the rectifying device.

Traditionally, AC-DC power converters have been realized using diode-based architectures such as the Greinacher cell, the Dickson multiplier [10], or a full-wave bridge rectifier [11]. Diode-based rectifiers have simply been realized in CMOS technology using diode-connected transistors such as the Dickson rectifier shown in Fig.2a. However, they suffer from poor sensitivity at low input power and from high dropout voltage that degrades their power conversion efficiency at midhigh input power. Enhanced sensitivity can be achieved either using Schottky diodes that require additional fabrication steps and hence are seldom offered in conventional CMOS processes or using integrated step-up transformer occupying large area [12] . On the other hand, the differential, fully cross-coupled (FX) rectifier [2], shown in Fig.2b, is widely used in RFID applications due to its improved sensitivity and high peak efficiency. However, this improvement is gained at the expense of the reverse characteristic of the rectifying devices, because the rectifying cross-coupled transistor is still a bidirectional

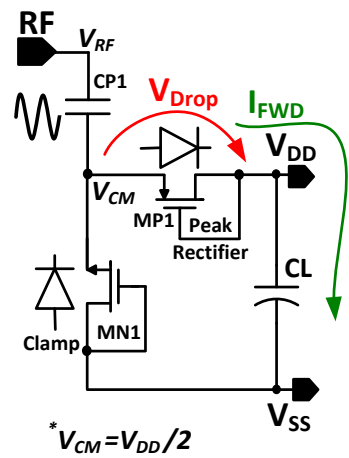

(a)

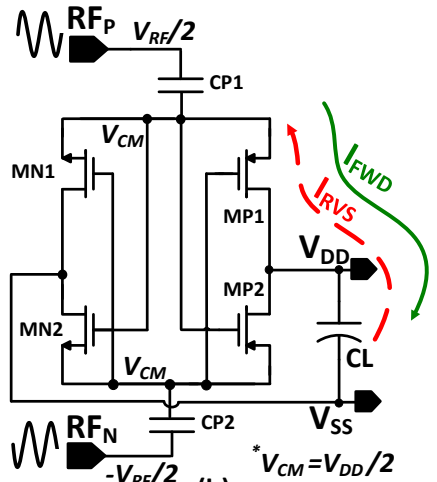

(b)
Fig. 2. (a) Schematic of Dickson rectifier and (b) Conventional FX rectifier. 


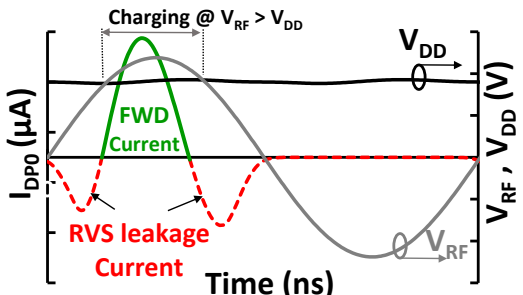

(a)

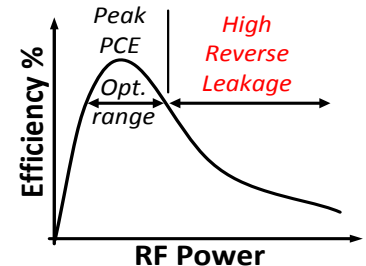

(b)
Fig. 3. (a) Conventional FX rectifier efficiency versus RF power and (b) its alternating current, output DC voltage, and RF voltage.

device—unlike diodes or diode-connected transistors. Hence, it conducts in a reverse direction once the instantaneous value of the RF signal, $V_{R F}$ becomes lower than the output DC voltage, $V_{D D}$, in every RF cycle, as shown in Fig.3a. This periodic reverse leakage is exacerbated as the RF power level grows, degrading the rectifier conversion efficiency at high input power, as shown in Fig.3b. Consequently, the rectifier operates efficiently within a limited range of input power. To evaluate different rectifier architectures, a dynamic range is defined as the input-power range at which the rectifier maintains PCE higher than $80 \%$ of its peak efficiency [13].

In [4], [5], a low $\mathrm{MHz}$ adaptive architecture reconfigures the rectifier stage as a diode-connected voltage doubler or as a half cross-coupled rectifier based on the input power level. However, it requires active diodes driven by fast comparators that are not power efficient at high RF frequencies such as UHF $433 \mathrm{MHz}$ or higher. A multistage configuration is presented in [13], [14] to enhance the efficiency of the cross-coupled rectifier by rewiring stages as a series or parallel multistage rectifier. Though this technique extends the efficiency of the cross-coupled rectifier over a wide range of input power, it requires multiple stages with high input capacitance and low input resistance, thus complicating the matching network design. In [15], an adaptive offset calibration technique is presented to compensate the threshold voltage, $V_{t h}$, in diodebased rectifiers to improve their sensitivity. However, it requires two more auxiliary, multistage rectifiers to compensate the $V_{t h}$ of the main rectifier, thus occupying a large area, adding more losses, and degrading the PCE.

In this paper, we propose and experimentally validate a self-biased, cross-coupled, differential CMOS rectifier with enhanced efficiency over wider input range than both diodeconnected and cross-coupled rectifiers. This facilitates reliable and efficient RF-to-DC power conversion at varying RF power levels, and it adds more spatial freedom between the wireless power transmitter and receiver. The proposed architecture is illustrated along with its operational concept in Section II, while the experimental results are discussed and compared with measurements of a fabricated conventional FX rectifier in Section III. Finally, the conclusion is drawn in Section V.

\section{Proposed RF-To-DC POWER CONVERTER}

\section{A. Proposed Architecture}

A schematic of the proposed self-biased cross-coupled rectifier is shown in Fig.4. The rectifier utilizes the crosscoupled configuration with the differential-drive capability to

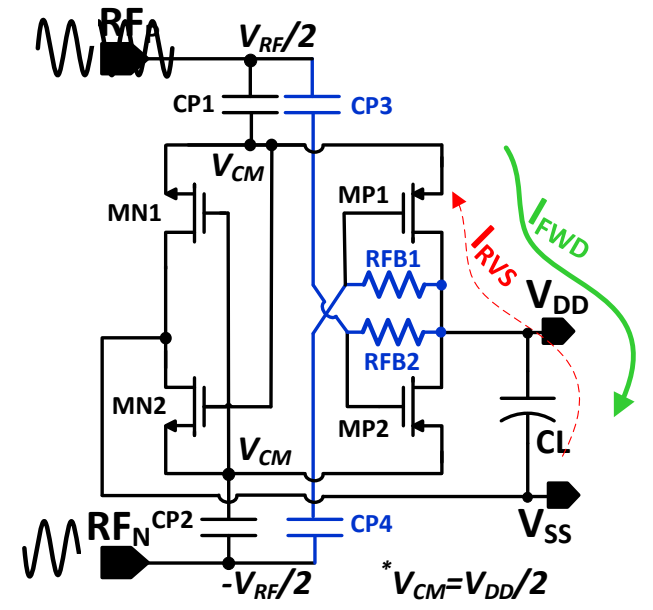

Fig. 4. Schematic of the proposed self-biased cross-coupled rectifier.

maintain good sensitivity at low input power by operating in the linear region and holding low dropout voltage. Moreover, a self-biasing mechanism is added to limit the reverse leakage at the sense of high input power. The proposed self-biasing mechanism controls the conduction of the rectifying devices by raising their effective turn-on voltage at high RF power levels. A simple implementation of this mechanism is achieved by applying the output $\mathrm{DC}$ voltage directly to the controlling gates of the rectifying transistors (MP1,2) without disturbing the RF signal at the differential inputs. This is achieved by decoupling the DC voltage of the rectifying PMOS MP1,2 gates apart of their corresponding NMOS MN1,2 using $(\mathrm{CP} 3,4)$ and then, applying the DC self-bias using an RF choke (RFC) coil that is DC short circuit and AC open circuit. It is worth noting that the self-biasing branch is connected to a MOSFET gate; hence, no DC current passes through it, whereas the self-biasing RFC branches can be simply replaced by high feedback resistors (RFB1, RFB2 $\simeq 100 k \Omega$ ) without loading the RF inputs, as shown in Fig.4.

\section{B. Operational Concept}

The operating point of the proposed self-biased crosscoupled rectifying device is compared to the conventional cross-coupled and diode-connected rectifying devices in Fig.5. Each rectifying device connects the AC-coupled $R F$ node (RF superimposed on a common mode $\left.V_{C M}=V_{D D} / 2\right)$ at its input terminal to the output $V_{D D}$ point. As shown in Fig.5a, the gate and drain of the diode-connected device are tied to the

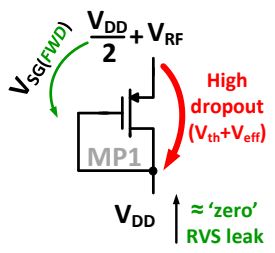

(a) Diode connected (Dickson multiplier)

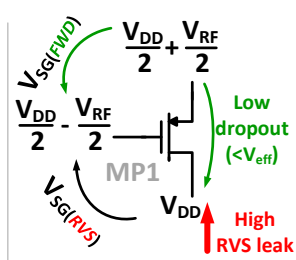

(b) Cross-coupled (Conventional)

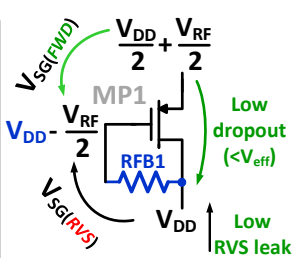

(c) Self-biased (Proposed)
Fig. 5. Operating point of the rectifying device (MP1) configured (a) as a diode-connected transistor (Dickson rectifier) (b) as a cross-coupled transistor, and (c) as a self-biased transistor (proposed rectifier). 


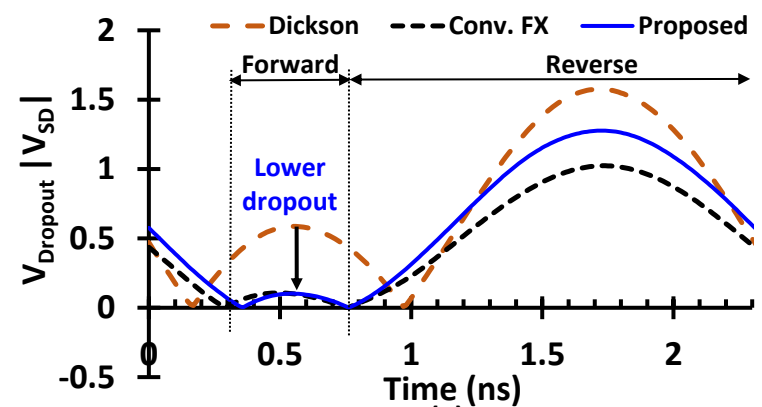

(a)

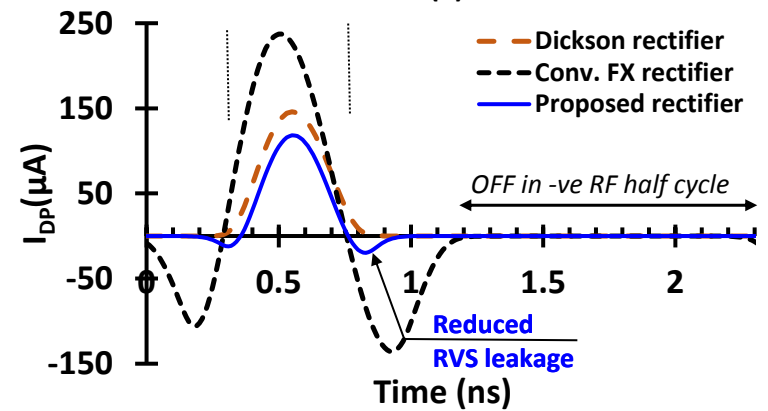

(b)

Fig. 6. Simulation of (a) the dropout voltage $\left(V_{S D}\right)$, and (b) drain current $\left(I_{D P}\right)$ in the rectifying PMOS devices of the Dickson, conventional FX, and proposed rectifiers at $37 \mu \mathrm{W}$ input power level.

output $V_{D D}$ to act as a two-terminal diode. In the case of the conventional cross-coupled device, the gate is attached to the $R F_{N}$ node $\left(=-V_{R F} / 2\right.$ superimposed on $\left.V_{D D} / 2\right)$, as shown in Fig.5b. However in the proposed self-biased cross-coupled device, the gate $R F_{N}$ signal is superimposed on $V_{D D}$, as shown in Fig.5c. Note that both the proposed self-biased crosscoupled device and the diode-connected device need minimum turn-on RF voltage, $V_{R F m i n}$, equal to threshold voltage, $V_{t h}$, plus $V_{D D} / 2$. This is unlike the conventional cross-coupled device, which requires $V_{t h}$ only. Moreover, the proposed device conducts in the linear region and holds a dropout voltage of less than or equal to its overdrive voltage, $V_{e f f}$, passing higher output voltage than the diode-connected device, which requires a dropout of $V_{t h}+V_{\text {eff }}$. As a result, the proposed self-biased cross-coupled device retains moderate sensitivity (i.e., it generates higher output voltage at the same $\mathrm{RF}$ input power) compared to the diode-connected device. This is derived from the operating points shown in Fig.5, and it matches the simulated dropout voltages of the three rectifiers at the same input power, as shown in Fig.6a.

During the periodic RF signal transitions, the reverse leakage of the diode-connected device is negligible, while the conventional cross-coupled device is strongly biased by $V_{S G(R V S)}=V_{R F} / 2+V_{D D} / 2$ in the reverse direction, as derived from Fig.5. Accordingly, the reverse current lobes increase, as shown in Fig.6b. On the other hand, as depicted in Fig.5, the proposed device is biased only at $V_{S G(R V S)}=$ $V_{R F} / 2$, leading to reduced reverse current lobes, as shown in Fig.6b. It worth noting that, during the negative RF half cycle, the gate of the MP1 in the conventional FX and proposed rectifiers is positively biased, shutting down its current completely, like the reverse-biased, diode-connected device.

Fig.7a shows the simulation of the output voltage versus the input power for all three rectifiers for a load of $50 k \Omega$. At

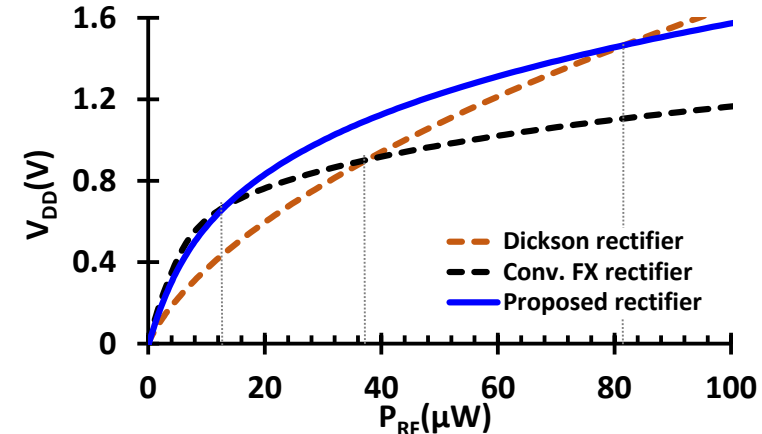

(a)
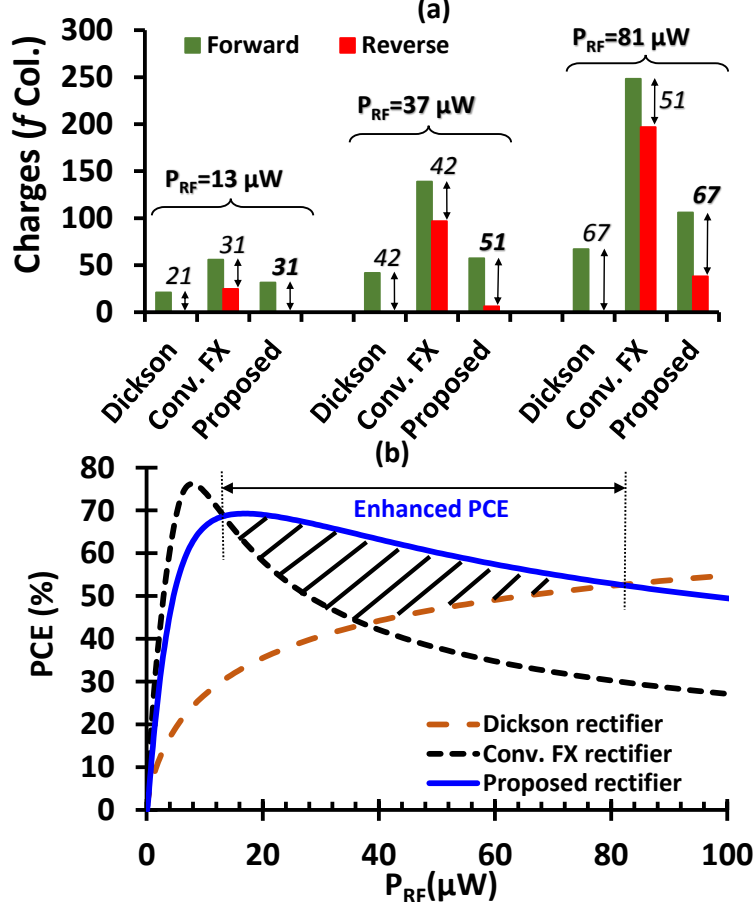

(c)

Fig. 7. (a) Simulated output voltage, (b) alternating charges flowing in MP1 per RF cycle (Qp), and (c) power conversion efficiency (PCE) for the Dickson, conventional FX, and proposed rectifiers versus RF input power.

low RF power, the proposed rectifier outperforms the Dickson rectifier while it outperforms the FX rectifier at high RF power. This can be explained by plotting both the forward harvested charges and the reverse leakage charges at three distinct RF power levels, as shown in Fig. 7b. The simulated PCE of the three rectifiers versus input power is plotted in Fig 7c. As shown in Fig. 7, the conventional FX rectifier has a peak PCE at low RF power, but it falls off rapidly due to its high reverse leakage current, which is shown in Fig.6b. On the other hand, the Dickson (diode-based) rectifier displays a moderate PCE at the high RF power levels but suffers from poor efficiency at low input power due to its high dropout voltage, shown in Fig.6a. Conversely, the proposed rectifier achieves wider dynamic range with a slight decrease in its peak PCE relative to the conventional FX rectifier.

A comparison of the three rectifying devices is summarized in Table I, where the proposed self-biased rectifying device shows competitive advantages of low dropout voltage, as shown in Fig.6a, and hence low dissipation loss and better sensitivity than the diode-connected device. At the same time, the proposed rectifying device shows lower reverse leakage 
TABLE I

COMPARISON OF RECTIFYING DEVICES

\begin{tabular}{|c|c|c|c|}
\hline Rectifier & Dickson & Conv. FX & Proposed \\
\hline Device & D-connected & X-coupled & Self-biased \\
\hline $\begin{array}{c}\text { Turn-on }{ }^{1} \\
\left(V_{\text {RFmin }}\right)\end{array}$ & $\begin{array}{c}\text { High } \\
\left(=\mathrm{V}_{\mathrm{DD}} / 2+\mathrm{V}_{\mathrm{th}}\right)\end{array}$ & $\begin{array}{l}\text { Low } \\
\left(=V_{\text {th }}\right)\end{array}$ & $\begin{array}{c}\text { High } \\
\left(=V_{\mathrm{DD}} / 2+\mathrm{V}_{\mathrm{th}}\right)\end{array}$ \\
\hline $\begin{array}{c}\text { Dropout }^{2} \\
\left(V_{\text {drop }}\right) \\
\end{array}$ & $\begin{array}{l}\text { High (Sat.) } \\
\left(=\mathrm{V}_{\mathrm{th}}+\mathrm{V}_{\mathrm{eff}}\right)\end{array}$ & $\begin{array}{c}\text { Low (Linear) } \\
\left(=\mathrm{V}_{\text {eff }}\right)\end{array}$ & $\begin{array}{c}\text { Low (Linear) } \\
\left(=V_{\text {eff }}\right)\end{array}$ \\
\hline Sensitivity $^{3}$ & Poor & Good & Moderate \\
\hline RVS leak & Negligible & High, Id Mos & Low \\
\hline $\begin{array}{c}\text { RVS } \\
\text { Leakage } \\
\text { Range }^{4} \\
\end{array}$ & $\begin{array}{l}\text { Negligible leak } \\
V_{R F}>V_{\text {breakdown }}\end{array}$ & $\begin{array}{c}\text { Wide leak } \\
2 \mathrm{~V}_{\mathrm{th}}-\mathrm{V}_{\mathrm{DD}}<\mathrm{V}_{\mathrm{RF}} \\
<\mathrm{V}_{\mathrm{RF}}<\mathrm{V}_{\mathrm{DD}}\end{array}$ & $\begin{array}{l}\text { Low leak } \\
2 V_{\text {th }}<V_{\text {RF }} \\
V_{\text {RF }}<V_{\text {DD }} \\
\end{array}$ \\
\hline \multirow{2}{*}{$\begin{array}{c}\text { Efficiency } \\
\text { (PCE) }\end{array}$} & Low peak & High peak & Good peak \\
\hline & Moderate-range & Narrow-range & Wide-range \\
\hline
\end{tabular}

and wider input range than the conventional cross-coupled device, as shown in Figs. $6 \mathrm{~b}$ and 7c, respectively.

\section{RESULTS AND DISCUSSION}

The proposed rectifier is implemented in a $0.18 \mu \mathrm{m}$ CMOS process technology, and occupies $130 \mu \mathrm{m} \times 130 \mu \mathrm{m}$ active area. The die microphotograph is shown in Fig.8. A conventional FX rectifier is implemented on the same die for a fair comparison to the proposed rectifier using identical test setup and conditions. The RF measurement setup includes Agilent's vector network analyzer (N5225A) and digital multimeter (34420A). The rectifier's power conversion efficiency (PCE) is measured with a single tone $433 \mathrm{MHz}$ signal at different input power levels, and the output DC voltage is recorded. After de-embedding the reflection and transmission losses, the net input power is calculated and the power conversion efficiency for the proposed and conventional rectifiers is plotted in Fig.9 versus the input power at different loads. Although the proposed rectifier has lower peak PCE $(\simeq 65 \%)$ than the conventional rectifier, whose peak $\mathrm{PCE} \simeq 75 \%$, the proposed architecture maintains its power conversion efficiency over a broader dynamic range of input power. Thus, the proposed rectifier can operate efficiently at different RF power levels, enabling robust wireless powering from varying transmission distances or within unstable environments.

To elucidate the relative PCE enhancement of the proposed rectifier, the ratio of the proposed rectifier PCE over the conventional FX rectifier PCE is plotted in Fig.10a for

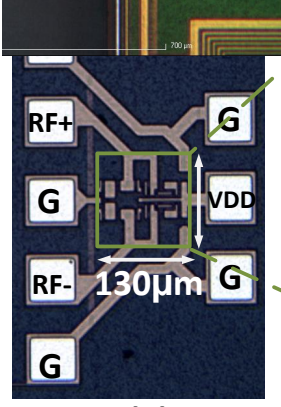

(a)

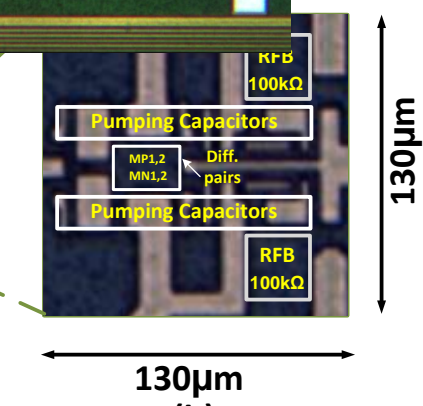

(b)
Fig. 8. Die microphotograph of (a) the proposed self-biased rectifier and (b) enlarged view of active area with the feedback resistors are highlighted.

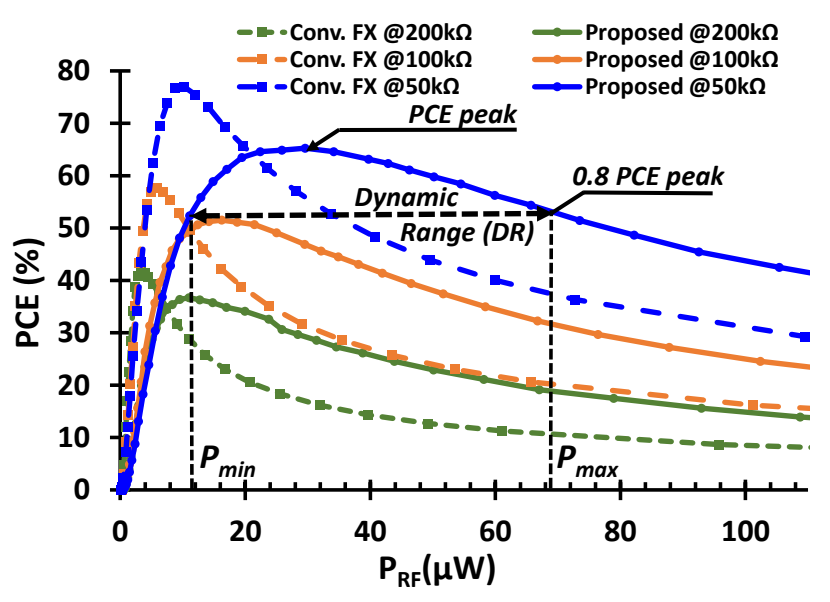

Fig. 9. Measured PCE of the proposed (solid) and conventional FX (dash) rectifiers versus input power for three loads.

variable loads $R_{L}=50 k, 100 k$, and $200 k \Omega$. An approximately $40 \%$ to $70 \%$ efficiency improvement is achieved in the proposed rectifier at mid-high input power $(>30 \mu W)$ relative to the conventional FX rectifier, as shown in Fig.10a. Moreover, the proposed rectifier maintains the achieved improvement of PCE over a wide input range at different loading conditions, as depicted in Figs. 9 and 10a. To further illustrate this point, the dynamic range of input power $\left[P_{\min }\right.$ to $\left.P_{\max }\right]$ at which the rectifier maintains $80 \%$ of its peak efficiency is represented as the ratio of $P_{\max }$ over $P_{\min }$ and is compared in Fig. 10b for the proposed and conventional FX rectifiers versus different loading conditions. Evidentially, the proposed

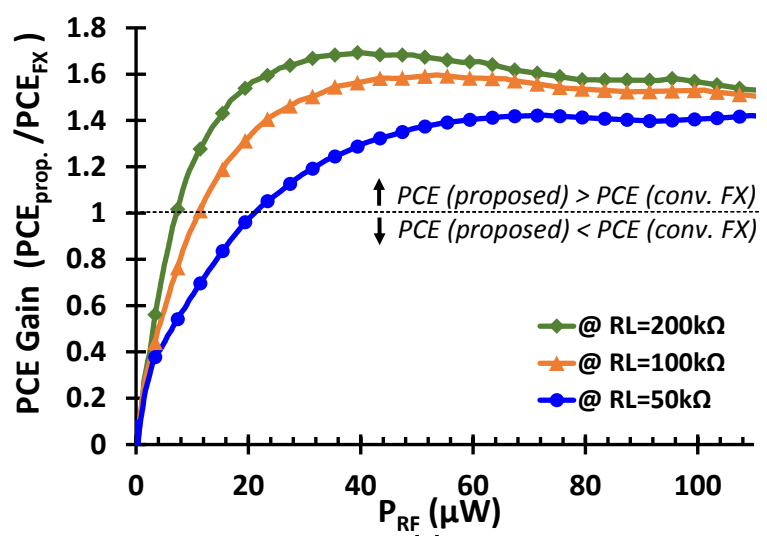

(a)

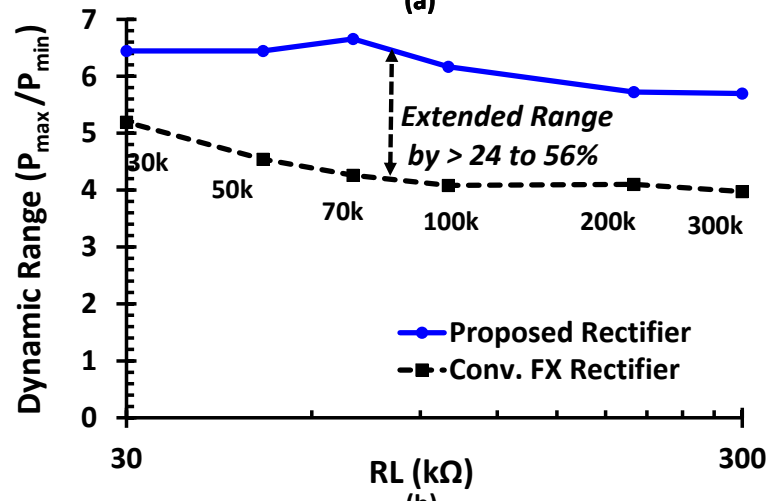

(b)

Fig. 10. (a) Measured ratio of the proposed rectifier PCE relative to the conventional FX rectifier PCE for three different loads, and (b) measured dynamic range of the proposed and conventional rectifiers. 
TABLE II

PERFORMANCE COMPARISON.

\begin{tabular}{|c|c|c|c|c|c|c|c|c|c|}
\hline Ref & $\begin{array}{l}\text { CMOS } \\
\text { Process }\end{array}$ & Architecture & \# stages & $\begin{array}{l}\text { Chip Area } \\
\left(m m^{2}\right)\end{array}$ & Frequency & $\begin{array}{l}\text { Sensitivity } \\
P_{R F} @ V_{o}=1 \mathrm{~V}\end{array}$ & $\begin{array}{l}\text { Load } \\
\left(R_{L}\right) \\
\end{array}$ & $\begin{array}{l}\text { Peak } \\
\text { PCE }\end{array}$ & $\begin{array}{c}\text { Dynamic Range }^{a} \\
P_{\max } / P_{\min } \\
\end{array}$ \\
\hline \multirow{2}{*}{$\begin{array}{l}\text { This } \\
\text { Work }\end{array}$} & \multirow{2}{*}{$0.18 \mu m$} & \multirow{2}{*}{$\begin{array}{l}\text { Adaptive self-biased cross- } \\
\text { connected rectifier (Proposed) }\end{array}$} & \multirow{2}{*}{ 1-stage } & \multirow{2}{*}{0.017} & \multirow{2}{*}{$433 \mathrm{MHz}$} & $-15.2 \mathrm{dBm}$ & $\mathbf{5 0} k \Omega$ & $65.3 \%$ & 6.5 \\
\hline & & & & & & $-17 \mathrm{dBm}$ & $100 k \Omega$ & $51.5 \%$ & 6.2 \\
\hline \multirow{2}{*}{$\begin{array}{l}\text { This } \\
\text { Work }\end{array}$} & \multirow{2}{*}{$0.18 \mu \mathrm{m}$} & \multirow{2}{*}{$\begin{array}{l}\text { Fully cross-coupled (FX) } \\
\text { rectifier (Conventional) }\end{array}$} & \multirow{2}{*}{1 -stage } & \multirow{2}{*}{0.005} & \multirow{2}{*}{$433 \mathrm{MHz}$} & $-13.6 \mathrm{dBm}$ & $50 k \Omega$ & $77 \%$ & 4.5 \\
\hline & & & & & & $-14.5 \mathrm{dBm}$ & $100 \mathrm{k} \Omega$ & $57.5 \%$ & 4.1 \\
\hline \multirow{2}{*}{ [6] } & \multirow{2}{*}{$0.18 \mu \mathrm{m}$} & \multirow{2}{*}{$\begin{array}{l}\text { Fully cross-coupled with } \\
\text { inter-stage RF injection }\end{array}$} & \multirow{2}{*}{3 -stage } & \multirow{2}{*}{0.088} & \multirow{2}{*}{$433 \mathrm{MHz}$} & $-7.8 \mathrm{dBm}$ & $30 k \Omega$ & $13.2 \%$ & 3.2 \\
\hline & & & & & & $-4.1 \mathrm{dBm}$ & $100 \mathrm{k} \Omega$ & $10 \%$ & 3 \\
\hline \multirow[b]{2}{*}{ [15] } & \multirow{2}{*}{$0.18 \mu \mathrm{m}$} & \multirow{2}{*}{$\begin{array}{l}\text { Dickson rectifier with multiple- } \\
V_{t h} \text { offset cancellation. }\end{array}$} & \multirow{2}{*}{ 4-stage } & \multirow{2}{*}{0.15} & \multirow{2}{*}{$433 \mathrm{MHz}$} & $-11.2 \mathrm{dBm}$ & $50 k \Omega$ & $32 \%$ & 4.2 \\
\hline & & & & & & $-14 \mathrm{dBm}$ & $100 \mathrm{k} \Omega$ & $34 \%$ & 4.7 \\
\hline \multirow[b]{2}{*}{ [16] } & \multirow{2}{*}{$0.18 \mu \mathrm{m}$} & \multirow{2}{*}{$\begin{array}{l}\text { Hybrid } V_{t h} \text { cancellation using } \\
\text { RF input \& DC output voltage }\end{array}$} & \multirow{2}{*}{ 1-stage } & \multirow{2}{*}{ NA } & \multirow{2}{*}{$433 \mathrm{MHz}$} & $>0 \mathrm{dBm}^{b}$ & $30 k \Omega$ & $12.5 \%$ & 3.3 \\
\hline & & & & & & $>0 \mathrm{dBm}^{b}$ & $100 \mathrm{k} \Omega$ & $4.6 \%$ & 3.6 \\
\hline
\end{tabular}

${ }^{a}$ Input power range of PCE $\geqslant 0.8$ Peak PCE.

${ }^{b} 0 \mathrm{dBm}$ for $V o=0.78 \mathrm{~V}$.

rectifier achieves a higher dynamic range across a wide range of loading conditions (from $30 k$ to $300 k \Omega$ ). Fig.11 depicts the measured output DC voltage versus input power level for the proposed and conventional FX rectifiers equally loaded by $50 k, 100 k$ and $200 k \Omega$. At the same input power, the proposed self-biased rectifier delivers higher DC output voltage than the conventional $\mathrm{FX}$ rectifier by $20 \%$ to $30 \%$.

A performance comparison of the proposed rectifier versus recent ISM $433 \mathrm{MHz}$ rectifiers is summarized in table II. The proposed rectifier shows $2 \times$ improvement in dynamic range and $5 \times$ enhancement in efficiency relative to [6]. More than $50 \%$ wider dynamic range and doubled efficiency is achieved compared to [15] with a $9 \times$ chip area saving.

\section{CONCLUSION}

In this paper, we propose a self-biased, cross-coupled, differential rectifier with $50 \%$ enhanced power conversion efficiency over a wide range of input power levels and under different loading conditions. A sensitivity of $\left(P_{R F}=30 \mu \mathrm{W}\right.$ at $V_{o}=1 \mathrm{~V}$ ) and peak power conversion efficiency (peak $\mathrm{PCE}=65.3 \%$ ) are achieved for a $50 k \Omega$ load. The architecture of the proposed rectifier is presented, and its performance is compared with the conventional, cross-coupled rectifier.

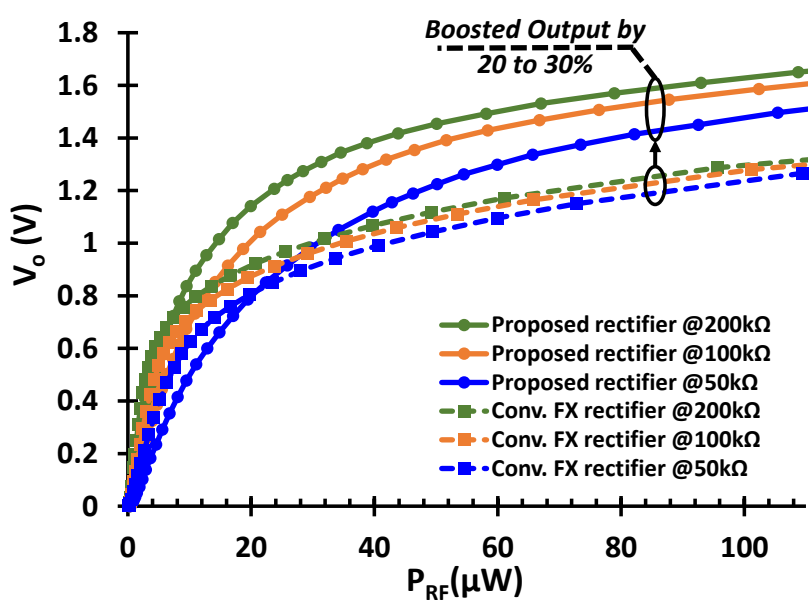

Fig. 11. Measured output DC voltage of the proposed and conventional FX rectifiers at $433 \mathrm{MHz}$ versus $\mathrm{RF}$ input power for various loads.

\section{REFERENCES}

[1] Y.-J. Kim, H. S. Bhamra, J. Joseph, and P. P. Irazoqui, "An ultralow-power RF energy-harvesting transceiver for multiple-node sensor application," IEEE Trans. Circuits Syst. II: Express Briefs, vol. 62, no. 11 , pp. $1028-1032$, Nov. 2015.

[2] K. Kotani, A. Sasaki, and T. Ito, "High-efficiency differential-drive CMOS rectifier for UHF RFIDs," IEEE J. Solid-State Circuits, vol. 44, no. 11 , pp. $3011-3018$, Nov. 2009.

[3] M. H. Ouda, M. Arsalan, L. Marnat, A. Shamim, and K. N. Salama, "5.2$\mathrm{GHz}$ RF power harvesting module in $0.18 \mu \mathrm{m}$ CMOS for biomedical implantable sensors," IEEE Trans. Microw. Theory Tech., vol. 61, no. 5, pp. 1-8, May 2013.

[4] X. Li, C. ying Tsui, and W.-H. Ki, "Power management analysis of inductively-powered implants with $1 \mathrm{X} / 2 \mathrm{X}$ reconfigurable rectifier," IEEE Trans. Circuits Syst. I, Reg. Papers, vol. 62, no. 3, pp. 617-624, March 2015.

[5] H.-M. Lee and M. Ghovanloo, "An adaptive reconfigurable active voltage doubler/rectifier for extended-range inductive power transmission," IEEE Trans. Circuits Syst. II: Express Briefs, vol. 59, no. 8, pp. 481 -485, Aug. 2012.

[6] S. S. Chouhan and K. Halonen, "A novel cascading scheme to improve the performance of voltage multiplier circuits," Analog Integr. Circ. Sig. Process, vol. 84, no. 3, pp. 373-381, 2015.

[7] A. A. Abdou, A. Shaw, A. Mason, A. Al-Shamma'a, J. Cullen, S. Wylie, and M. Diallo, "A matched Bow-tie antenna at $433 \mathrm{MHz}$ for use in underwater wireless sensor networks," J. of Physics: Conf. Series, vol. 450, no. 1, p. 012048, 2013.

[8] J. Zhu, L. Wu, X. Zhang, C. Jia, and C. Zhang, "A low-power $433 \mathrm{MHz}$ transmitter for battery-less tire pressure monitoring system," in IEEE 9th International Conf. on ASIC (ASICON), Oct. 2011, pp. 184-187.

[9] S. H. Abdelhalem, P. S. Gudem, and L. E. Larson, "An RF-DC converter with wide-dynamic-range input matching for power recovery applications," IEEE Trans. Circuits Syst. II: Express Briefs, vol. 60, no. 6, pp. 336-340, June 2013.

[10] J. Dickson, "On-chip high-voltage generation in MNOS integrated circuits using an improved voltage multiplier technique," IEEE J. SolidState Circuits, vol. 11, no. 3, pp. 374 - 378, June 1976.

[11] S. Dwari, R. Dayal, L. Parsa, and K. N. Salama, "Efficient direct acto-dc converters for vibration-based low voltage energy harvesting," in IEEE 34th Industrial Electronics Conf. (IECON), 2008., Nov. 2008, pp. $2320-2325$

[12] H. Goncalves, M. Martins, and J. Fernandes, "Fully integrated energy harvesting circuit with -25-dBm sensitivity using transformer matching," IEEE Trans. Circuits Syst. II: Express Briefs, vol. 62, no. 5, pp. 446-450, May 2015.

[13] C.-J. Li and T.-C. Lee, "2.4-GHz High-efficiency adaptive power harvester," IEEE Trans.VLSI Syst., vol. 22, no. 2, pp. 434-438, Feb 2014.

[14] L. . B. A. Scorcioni, S. ; Larcher, "A reconfigurable differential CMOS RF energy scavenger with $60 \%$ peak efficiency and $-21 \mathrm{dBm}$ sensitivity," IEEE Microw. Wireless Compon. Lett., 2013.

[15] K. Gharehbaghi, O. Zorlu, F. Kocer, and H. Kulah, "Auto-calibrating threshold compensation technique for RF energy harvesters," in IEEE RFIC Symposium, May 2015, pp. 179-182.

[16] S. S. Chouhan and K. Halonen, "Threshold voltage compensation scheme for RF-to-DC converter used in RFID applications," Electron. Lett., vol. 51, no. 12, pp. 892-894, 2015. 